encourage screening for established cerebrovascular risk factors in this high-risk, vulnerable group.

\section{OP100 THE COST-EFFECTIVENESS AND EQUITY OF THE NHS HEALTH CHECKS CARDIOVASCULAR DISEASE PREVENTION PROGRAMME: A MICROSIMULATION USING REAL-WORLD DATA FROM A DEPRIVED NORTHERN CITY}

${ }^{1} \mathrm{~B}$ Collins*, ${ }^{1} \mathrm{C}$ Kypridemos, ${ }^{2} \mathrm{P}$ Parvulescu, ${ }^{2} \mathrm{R}$ Gosling, ${ }^{1} \mathrm{~S}$ Capewell, ${ }^{1} \mathrm{M}$ O'Flaherty. ${ }^{1}$ Department of Public Health and Policy, University of Liverpool, Liverpool, UK; ${ }^{2}$ Public Health Department, Liverpool City Council, Liverpool, UK

\subsection{6/jech-2017-SSMAbstracts.99}

Background The NHS Health Check Programmes' stated objective is the early identification of otherwise healthy people at high risk of cardiovascular disease (CVD) and diabetes. However, the programme's effectiveness, cost-effectiveness, and equity are still contested. This study therefore aimed to determine whether Health Checks (HCs) are cost-effective and equitable in a city with high levels of deprivation and CVD.

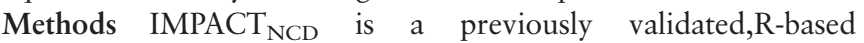
dynamic stochastic microsimulation policy model. We calibrated this model to Liverpool demographics, risk factor exposures, and CVD epidemiology. We modelled the current implementation of HCs using local and national data on effectiveness, costs, and participant risk profiles. Disease costs and health state utilities were drawn from standard sources and discounted at $3.5 \%$ annually using a healthcare perspective. We quantified the uncertainty of model outputs using secondorder Monte Carlo simulation, and report 95\% uncertainty intervals.

We modelled three fifteen year scenarios from 2017 to 2031:

A) continuing the current implementation of HCs;

B) an optimal implementation of HCs assuming optimal coverage, uptake, treatment and lifestyle change;

C) combining scenario A with structural policies targeting dietary consumption of salt, sugar, fruit and vegetables.

We compared all three scenarios with a counterfactual of no HCs, and conducted a rigorous sensitivity analysis.

Results The model suggested that over 15 years the CVD cases prevented or postponed would be approximately 310 (40-734) for scenario A, $870(327-1,397)$ for scenario B, and $1740(815-2,939)$ for scenario C.

Cumulative discounted net costs and quality-adjusted life years (QALYs) gained for the three scenarios respectively would be $+£ 2.1 \mathrm{~m}(£ 1.5 \mathrm{~m}-+£ 4.8 \mathrm{~m})$ and +90 QALYs $(-124-+376)$ for $\mathrm{A} ; \quad+£ 1.4 \mathrm{~m} \quad(£ 6.1 \mathrm{~m}-+£ 6.6 \mathrm{~m})$ and +434 QALYs $(-76-+1,133)$ for $\mathrm{B}$; or $£ 16.9 \mathrm{~m}$ $(£ 33.2 \mathrm{~m}-£ 5.9 \mathrm{~m})$ and $+2,871$ QALYs $(+1,355-+4,830)$ for $\mathrm{C}$.

We estimated the probability of scenarios $\mathrm{A}$ and $\mathrm{B}$ being cost-effective by 2031 at $25 \%$ and $74 \%$ respectively, valuing each QALY at $£ 20000$.

Scenario C would become cost saving by 2030 .

Scenario A may increase existing health inequalities; $\mathrm{B}$ is likely to be neutral, while $\mathrm{C}$ would substantially decrease inequalities.

In extensive sensitivity analyses, the direction of the results did not change when the discount rate was varied from $0 \%-$ $6 \%$, nor when the time horizon was increased to 20 years.
Conclusion Current NHS Health Checks implementation appears neither equitable nor cost-effective. The addition of structural policies proved equitable and cost saving. Future research might now seek to identify the optimal combination of structural policies at local level.

\section{SSM annual scientific meeting 2017}

\section{Plenary $(\mathrm{PL})$ presentations}

\section{PL01 THE IMPACT OF CO-LOCATED WELFARE ADVICE IN PRIMARY HEALTHCARE SETTINGS ON MENTAL HEALTH AND HEALTH SERVICE USE: A MIXED METHODS EVALUATION}

${ }^{1} \mathrm{C}$ Woodhead*, ${ }^{2} \mathrm{M}$ Khondoker, ${ }^{1} \mathrm{R}$ Raine. ${ }^{1}$ Applied Health Research, University College London, London, UK; ${ }^{2}$ Medicine and Health Sciences, University of East Anglia, Norwich, UK

\subsection{6/jech-2017-SSMAbstracts. 100}

Background Co-locating welfare advice services in primary healthcare settings has been one approach to tackling health inequalities by increasing income among socially deprived individuals. It is also hoped to relieve pressure on general practices in supporting patients with 'non-clinical' needs. Previous evaluations have been methodologically limited and lack theoretical underpinning. We aimed to examine the impact of colocated welfare benefits and debt advice on mental health and primary care service use, and to develop theory linked to pathways of effect.

Methods A prospective, controlled quasi-experimental study with an embedded qualitative component was carried out (December 2015-December 2016) in eight intervention and nine comparator sites across North Thames, London. Beforeand-after quantitative data were collected via self-report questionnaires. Comparison group members were propensity score weighted for analyses. Outcomes included change in symptoms of common mental disorder (CMD) (12-item General Health Questionnaire), well-being (Shortened Warwick and Edinburgh Mental Well-being Scale), three-month GP consultation rate and financial strain. Data from qualitative interviews with 24 primary care staff, funders and advice providers were analysed using a modified realist evaluation approach to understand how co-located welfare advice could influence practice outcomes.

Results For the quantitative study, $n=285$ and $n=633$ individuals were recruited into advice and comparison groups respectively at baseline. $72 \%$ and $84 \%$ were retained at 3 month follow-up. Relative to controls, CMD caseness reduced significantly among female and Black/Black British advice recipients. Individuals whose advice resulted in positive outcomes demonstrated significantly improved well-being scores. Significant reduction in financial strain overall but no change in threemonth consultation rate was found. Per capita, advice recipients received $£ 15$ per $£ 1$ of funder investment. Qualitative findings were used to inform underlying theory linking service activity to general practice outcomes. These were reduced GP consultations for 'non-clinical' issues and reduced practice staff time supporting patients with such issues. The findings revealed key implementation, context and agency factors that facilitated or hindered the potential for co-located advice to influence these outcomes. 